\title{
Chronological Age and Training Age as Determinants of Soccer Specific Speeds
}

Received $23^{\text {rd }}$ August 2019 Accepted $26^{\text {th }}$ October 2019 www.ijpefs.com

\section{Belayneh Chekle a, ${ }^{*}$, Tefera Tadesse ${ }^{b}$ and Zerihun Birhanu a}

Abstract: The main purpose of this study was to unveil how chronological and training age or maturity of soccer players relate or affect linear sprinting speed, repeated sprinting ability (RSA), and change-ofdirection speed (CODS) performance. Competitive soccer players at the Ethiopian national soccer league level participated in the study. A total of 88 volunteered soccer players (age, $22.25 \pm 2.27$ years old; training age; $9.38 \pm 2.78$ years) who were free from any kind of injury at the time of data collection completed the study protocol. While age was calculated using a player's birth certificate, fitness performance was measured using specific test protocols for each fitness element. For credibility, fitness test was conducted on a weekly basis for about five consecutive weeks and the average was taken. Bivariate correlation, partial correlation and GLM analyses were used. The bivariate correlation showed that sprinting speed ( $\mathrm{r}(88)=-$ $.254, \mathrm{p}=.017)$, CODS ( $\mathrm{r}(88)=-216, \mathrm{p} .043$ ) and RSA best ( $(88)=0.235, \mathrm{p}=.028$ ) significantly correlated with age $(\mathrm{p}<.05)$. However, RSA total time, RSA average and RSA worst did not correlate with age. With training age, only CODS showed small, but significant negative correlation ( $\mathrm{r}(88)=-.230, \mathrm{p}=.031$ ). While sprinting speed significantly correlated with maturity, other qualities that rely on the ability to produce the best performance repeatedly did not relate with age or training age. These findings suggest that speed and speed related performances depend on maturity and genetic make-up and these are less trainable qualities that may not significantly improve over time.

Key Words: Age, Maturity, Speed, repeated sprinting ability, fitness

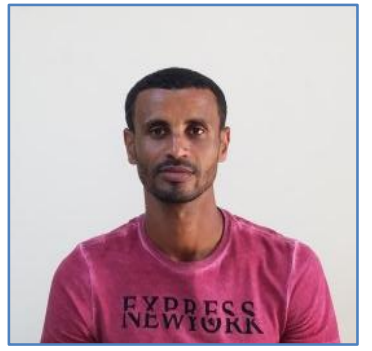

Belayneh Chekle (assistant professor) is a lecturer at Bahir Dar University and is at the verge defending his $P h D$ dissertation at Bahir Dar Unversity. Belayneh Chekle has received his Master's degree in football coaching from Addis Ababa University, Ethiopia. He was following hid PhD study for the last five years and waiting to defend his dissertation at Bahir Dar University, Ethiopia. He is also working as a coach of youth footballers in the sport Academy of Bahirdar University. Since 2017 he is an assistant professor in sport science department. His main research interest is on soccer performance, performance factors, fitness development of junior and elite players and health and exercise

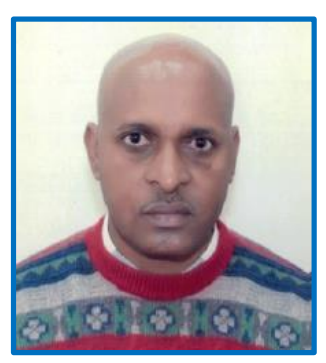

Tefera Tadesse Ph.D. is a Fulbright Postdoctoral Fellow in the Department of Counseling, Educational Psychology, and Special Education at Michigan State University, USA. Dr. Tadesse received his Ph.D. in Curriculum and Instruction from the School of Education in the University of Queensland, Australia. His major research interests are in higher education teaching and learning, student engagement, classroom instruction, cooperative learning, equity and social justice in education, sports sciences, and health sciences. 


\section{Introduction}

Performance improvement in soccer is the prime goal sought by coaches, players, and others around soccer clubs or teams $[1,2]$. The realization of a peak performance and the sacrifice for its maintenance is the ultimate underlying reason for coaches and players to work hard in today`s soccer [3]. This is most needed for winning or success, because now a day`s success and winning in soccer are a matter of big business. Though there is a greater emphasis placed on winning, soccer as a sport is too demanding [4,5]. Barnes et al., (2014) indicated that the game is becoming more demanding in terms of high-intensity running distance, number of high intensity actions, sprint distance and number of sprints [4]. Also, it is demanding physically requiring not only simple sprinting speed, endurance or strength, but also speed in different distances from $5 \mathrm{~m}$ to $60 \mathrm{~m}$ of 40 60 sprints with a short recovery in between $[6,7]$. It is becoming more demanding in terms of technical proficiency [8] that requires the most difficult skills $[9,10]$. Most importantly soccer requires the execution of technical skills explosively or at high tempo [11].

It is showed that technical-tactical ability is the distinguishing factor of successful teams from unsuccessful teams $[4,12]$, but without fitness it deteriorates $[11,13,14]$. This highlights the necessity of specific fitness development and maintenance in soccer. When fitness decays, technical-tactical proficiency and psychological vitality can be flooded away. This invaluable role of fitness for soccer success elicited teams to have a fitness coach, a nutritionist, strength and conditioning specialist, and a physiotherapist. Even those world class players pay a due regard to their soccer specific fitness. Therefore, competing at high level soccer demands players to have well developed physical qualities which can enable them to shine and utilize their technical-tactical qualities that the game demands. For example, strength, speed, and repeated sprinting ability (RSA) are important fitness components to combat and win the limited ball contacts encountered during match play $[8,12]$. All these signify the critical value of speed and speed related fitness has in soccer.

Soccer is known to its specific fitness requirements. For example, agility, acceleration, deceleration, speed and RSA are considered as critical components of soccer. For this, soccer is an intermittent sport with numerous short, explosive, high intensity bouts as sprinting, change of direction and jumping interspersed with short recovery periods over 90 minutes $[5,6]$. This is because that during matches players are expected to accelerate, decelerate, sprint and change direction throughout the game $[13,15,16]$. Moreover, time-motion analysis indicated that short distance sprint occurs more frequently during soccer matches $[5,7,17,13]$. Players perform 150-250 intense anaerobic actions during a game $[6,18]$. The capacity of soccer players to produce varied high-speed actions can greatly impact match performance. Although high-speed actions (anaerobic activities) account only approximately $11 \%$ of the total distance covered in a match they constitute the most crucial moments of the game, that is, mainly the difference between losing or winning ball possession, scoring or missing, saving or clearing and conceding goal [13]. For example, linear or straight sprinting is the most common high-speed action before those crucial moments during games [13]. Therefore, it is too logical to invest a lot or to study this segment of soccer fitness (i.e., sprinting speed and RSA), from different perspective.

Generally, those fitness parameters as speed, change-of-direction-speed and repeated sprinting ability (RSA) are the most important fitness qualities that modern soccer players expected to have. A lot of training protocols to improve and maintain these qualities are devised. For example, short term protocols of both complex (OCD plus plyometric) training protocol and isolated COD protocol are important to have meaningful improvements in speed parameters of soccer players [19, 20]. However, being in the same culture (football training \& style) and being in the same squad players do not have the same level of performance in these specific qualities. Also the response they show for the same training season or protocol is not 


\section{Belayneh Chekle et al., /2019}

affecting the same level. Therefore, the performances of soccer players in this regard need to be accounted for some other factors as maturity or age. This study, as a result came across with the relationship of age with these qualities and how maturity determines speed and speed related performance of soccer players. The main objectives of the study were to (i) identify how chronological age and training age relates linear sprinting speed, CODS and RSA performance in soccer and (ii) Reveal how maturity and training age determine soccer players' performance in 40m linear speed, 9-3-6-3-9 CODS and 6*35m RSA.

\section{Method}

The study used a correlational design so as to disclose the relationship of age or maturity and training age with speed, CODS, and RSA performance. A total of 88 outfield soccer players from five randomly selected teams participated in the study. 5 teams were selected randomly from 11 teams who were participating or competing in the national league. This league is the third league level in the nation next to the premier league and super league. The data regarding fitness performance was collected using valid and specific test protocols.

For measuring linear sprinting speed, 40-m linear sprinting was measured. Three trails for each player were given and the best score was taken for analysis (i.e., 7-9 minutes with standing passing as an active recovery between trials). For CODS, Concerning which agility tests are the most valid for the planned agility of soccer players, Sporis et al. (2010) found that, sprint 9-3-6-3-9 $\mathrm{m}$ with backward and forward running (SBF) is among the most valid and reliable tests (i.e., $\alpha=0.949$ ) [21]. As a result, this test has been used in this research to measure planned agility performance of the players. The distance the player has to cover was $24 \mathrm{~m}$ with forward sprinting and $6 \mathrm{~m}$ with backward running. Most sprints in soccer game are linear but, the test that I have selected to use better goes in parallel with this fact that the actions do not involve 90- or 180-degree sharp turn.
RSA performance was measured using the $35 \mathrm{~m} * 6$ test protocol. Cones have been set out at the end of a 35-meter running lane. Two testers were involved, as one person was required to time the 25 second recovery period. The player stands at one end of the $35 \mathrm{~m}$ lane and starts a maximal sprint on the command "go". Players were encouraged verbally to enable them produce maximum sprint through the distance. Then, after 25 seconds of recovery the next sprint starts from the opposite end of the $35 \mathrm{~m}$ lane. The sprint repeated the same way until 6 sprints were completed. Previous researches showed that, this test can be used as a valid and reliable method [22]. Furthermore, a recent study has also confirmed that running based anaerobic test (RAST) is a valid and reliable measure [23]. In relation with its validity, the nature of soccer is related to the test protocol and this alone can make the test relevant. This is because that a good sport specific test should be derived from logical reasoning, based on game analysis [24].

These tests were conducted for about five consecutive weeks and the averaged value has been taken for analysis. This was done to insure a more reliable data about the players' performance. The analysis was done using bivariate correlation, partial correlation and general linear model (GLM).

\section{Result and Discussion}

Basic information regarding the age, training age and bodily measurements is provided here under. They were $22.25 \pm 2.27$ years old with a training age of $9.30 \pm 2.78$ year's soccer training. They weighted $63.78 \pm 5.33 \mathrm{~kg}$ with a height of $172.91 \mathrm{~cm}$ tall (table 1).

Training age, which was measured in the number of years the players' participated in structured soccer training and soccer specific fitness was examined to reveal about how they relate. Fitness was assessed using those specific parameters as linear sprinting speed measured over 40-m dash, CODS (9-3-6-3-9) and RSA as measured with 35-m*6 test protocol 
Belayneh Chekle et al., /2019

Table 1 Basic Demographic Information of the Participants

\begin{tabular}{lrrrrr}
\hline & $\mathrm{N}$ & Minimum & Maximum & Mean & $\begin{array}{c}\text { Std. } \\
\text { Deviation }\end{array}$ \\
\hline Age & 88 & 18.00 & 30.00 & 22.2500 & 2.27050 \\
Training Age & 88 & 4.00 & 18.00 & 9.3864 & 2.78102 \\
Weight & 88 & 52.00 & 74.50 & 63.7830 & 5.33171 \\
Height & 88 & 150.00 & 185.00 & 172.9091 & 6.28744 \\
\hline
\end{tabular}

Table 2 The relationship of training age with fitness

\begin{tabular}{llrrrrrr}
\hline & & Speed & CODS & $\begin{array}{c}\text { RSA } \\
\text { Total } \\
\text { Time }\end{array}$ & $\begin{array}{r}\text { RSA } \\
\text { Average }\end{array}$ & $\begin{array}{c}\text { RSA } \\
\text { Best }\end{array}$ & $\begin{array}{c}\text { RSA } \\
\text { Worst }\end{array}$ \\
& & & & & & \\
\hline \multirow{3}{*}{ Training } & Pearson & -.188 & - & -.135 & -.130 & -.120 & -.118 \\
Age & Correlation & & $.230^{*}$ & & & & \\
& Sig. (2-tailed) & .080 & .031 & .208 & .228 & .265 & .274 \\
& $\mathrm{~N}$ & 88 & 88 & 88 & 88 & 88 & 88 \\
\hline
\end{tabular}

Table 3 The relationship between chronological age and soccer specific fitness

\begin{tabular}{rlrrrrrr}
\hline & Speed & CODS & $\begin{array}{c}\text { RSA } \\
\text { Total } \\
\text { Time }\end{array}$ & $\begin{array}{c}\text { RSA } \\
\text { Average }\end{array}$ & $\begin{array}{c}\text { RSA } \\
\text { Best }\end{array}$ & $\begin{array}{c}\text { RSA } \\
\text { Worst }\end{array}$ \\
\hline \multirow{3}{*}{ Age } & Pearson & $-.254^{*}$ & $-.216^{*}$ & -.208 & -.206 & $-.235^{*}$ & -.176 \\
& Correlation & & & & & & \\
& Sig. (2-tailed) & .017 & .043 & .052 & .055 & .028 & .100 \\
& $\mathrm{~N}$ & 88 & 88 & 88 & 88 & 88 & 88 \\
\hline
\end{tabular}

Table 4 The partial correlation between chronological age and fitness

\begin{tabular}{|c|c|c|c|c|c|c|c|}
\hline & & Speed & CODS & $\begin{array}{l}\text { RSA Total } \\
\text { Time }\end{array}$ & $\begin{array}{c}\text { RSA } \\
\text { Average }\end{array}$ & RSA Best & RSA Worst \\
\hline \multirow{3}{*}{ Age } & Correlation & -.167 & .033 & .092 & -.108 & .060 & .100 \\
\hline & $\begin{array}{l}\text { Significance (2- } \\
\text { tailed) }\end{array}$ & .132 & .769 & .408 & .331 & .591 & .368 \\
\hline & df & 81 & 81 & 81 & 81 & 81 & 81 \\
\hline
\end{tabular}




\section{Belayneh Chekle et al., /2019}

Training age does not have a significant relationship with linear sprinting speed or RSA performance of the players. What the basic physiological knowledge reminds is that speed more rely on somatotype (muscle fiber). Those with a fast twitch muscle fiber are the one innate for speed. Thus, speed can be seen as a fitness quality which is training resistant. The effect of training may not be as significant as heredity. But, CODS has showed a statistically significant positive relationship with training age/years $r(88)=-.208, p=.031$. Here the relationship tells us that a unit change in training year can result 0.208 second reduction in the 9-3-63-9 CODS test. Going in parallel, Nerga et al. (2018) showed that specific kind of training can result a significant improvement in CODS [20]. The perception and decision element present here with CODS can be impacted by training, though the speed element may not be impacted. CODS do not involve sheer speed, instead it demands on perceiving the space and controlling ones movement to change direction.

The selected soccer specific fitness parameters were also assessed about how they correlate with maturity level of the players. A zeroorder correlation test was used to identify how significantly maturity is related with soccer specific fitness.

Linear sprinting speed significantly correlated with chronological age of the players $r$ $(88)=-.254, p=.017$. As players get matured sprinting speed is expected to increase. Basically, maturity is associated with quality differentiation. Most importantly, with men's maturity can result hypertrophy and more testosterone production which can impact strength. Still the significant correlation between age and RSA best ( $\mathrm{r}(88)=-$ .235 , $\mathrm{p}<.028$ ), is an indicator that maturity related changes can heavily impact speed. Here the relationship of age with RSA average, RSA total and RSA worst time to be non-significant is an indicator that maturity impacts strength or speed mainly no other qualities which rely on aerobic fitness (RSA average, RSA total and RSA worst). These RSA measures depend highly on the players' ability to recover during the brief recovery times for subsequent sprints. The same way, maturity is significantly related with CODS ( $\mathrm{r}(88)=-.216$, $\mathrm{p}=.043$ ). Recent findings on the matter came across with similar findings. For example, speed and CODS were found to be better with more matured players [25]. Moreover, U23 players were found to be significantly faster than U16 players in 20-m linear sprinting speed (2.09 versus 2.98 seconds, $\mathrm{p}=0.02$; $E S=0.94$ ) (Bishop et al., 2019). Still other recent finding witnessed that repeated-sprint sets (RSS) improve with maturation in young soccer players $[26,27]$. Generally, while linear sprinting speed differs across ages [25], CODS performance level significantly differs across different chronological age and experience (training age) [25, 28].

Though, the zero-order correlation showed the relationship of age with neuromuscular fitness without considering the moderating effect the variables on the relationship nature, partial correlation was used to examine how each variable affects the relationship of age with specific fitness.

The fitness elements as speed, CODS and RSA are highly related. Here the partial correlation result indicated that the nature of the relationship between maturity and each fitness qualities was highly affected or moderated by each other. For this reason, the significant relationship found in the zero order correlation (table 3 ) is not found in the partial correlation test (table 4).

Using GLM, the overall regression model was found significant $F(1,86)=5.93, p=.017$. From this analysis it was found that $6.5 \%$ of the variance in linear sprinting speed can be explained by maturity. The other way, by determining the coefficient of determination from the correlation coefficient, we can have the same value that is 0.065 (6.5\%). From this, it seems that $80-90 \%$ speed performance can be accounted to heredity and the remaining to age/maturity (6.5\%) and remaining may be due to training and nutrition. The coefficient revealed that a year increment in age can result a 0.038 seconds reduction in the time to be taken to cover the $40-\mathrm{m}$ dash. Even though this amount of time change in 40 $\mathrm{m}$ sprinting is not negligible as a performance factor, sprinting speed is mostly left to genetic make-up, meaning that it is not such a trainable attribute. 
Table 5 the GLM of speed from age

\begin{tabular}{|c|c|c|c|c|c|c|}
\hline \multicolumn{7}{|c|}{ Dependent Variable: Speed } \\
\hline Source & $\mathrm{B}$ & $\mathrm{df}$ & Mean Square & $\mathrm{F}$ & Sig. & Partial Eta Squared \\
\hline Corrected Model & & 1 & .657 & 5.930 & .017 & .065 \\
\hline Intercept & 6.529 & 1 & 38.221 & 344.854 & .000 & .800 \\
\hline Age & -.038 & 1 & .657 & 5.930 & .017 & .065 \\
\hline Error & & 86 & .111 & & & \\
\hline Total & & 88 & & & & \\
\hline Corrected Total & & 87 & & & & \\
\hline
\end{tabular}

a. R Squared $=.065$ (Adjusted R Squared $=.054$ )

Table 6 GLM CODS from age

\begin{tabular}{lcccccc}
\hline Dependent Variable: & CODS & \multicolumn{7}{l}{} \\
\hline Source & $\mathrm{B}$ & $\mathrm{df}$ & Mean Square & $\mathrm{F}$ & Sig. & Partial Eta Squared \\
\hline Corrected & & 1 & 4.336 & 4.227 & .043 & .047 \\
Model & & & & & & \\
Intercept & 12.133 & 1 & 132.009 & 128.710 & .000 & .599 \\
Age & -.098 & 1 & 4.336 & 4.227 & .043 & .047 \\
Error & & 86 & 1.026 & & & \\
Total & & 88 & & & & \\
Corrected Total & & 87 & & & & \\
\hline
\end{tabular}

a. R Squared $=.047$ (Adjusted R Squared $=.036$ )

Table 7 GLM of RSA best from chronological age

\begin{tabular}{lcccccc}
\hline Dependent Variable: & RSA Best & & & & & \\
\hline Source & $\mathrm{B}$ & $\mathrm{df}$ & Mean Square & $\mathrm{F}$ & Sig. & $\begin{array}{c}\text { Partial Eta } \\
\text { Squared }\end{array}$ \\
\hline $\begin{array}{l}\text { Corrected } \\
\text { Model }\end{array}$ & & 1 & .397 & 5.012 & .028 & .055 \\
$\begin{array}{l}\text { Intercept } \\
\text { Age }\end{array}$ & 5.679 & 1 & 28.922 & 365.416 & .000 & .809 \\
Error & -.030 & 1 & .397 & 5.012 & .028 & .055 \\
Total & & 86 & .079 & & & \\
Corrected Total & & 88 & & & & \\
\hline
\end{tabular}

a. R Squared $=.055$ (Adjusted R Squared $=.044$ )

As a result, the obtained capacity of age to The overall regression model to predict CODS from explain speed is convincing and significant. The age is significant $F(1,86)=4.227, p<.001$. Maturity model can be summed up as follows: Speed $=6.529$ - level was found to account $4.7 \%$ of the variance in 9 0.038 (Age) or $\mathrm{Y}=6.529-0.038(\mathrm{X} 1)$

The prediction capacity of chronological age or maturity to CODS performance of soccer players has also been tested using GLM. 3-6-3-9 CODS performance. The same value can be found by using coefficient of determination from the zero-order correlation. This means that a unit change (increment) in age can cause a 0.098 seconds reduction in the time to cover the test. This magnitude effect of maturation on change-of- 


\section{Belayneh Chekle et al., /2019}

direction-speed performance is not negligible. This kind of fitness has such a positive relationship with linear sprinting speed, meaning that it is training resistant quality. On the other side, the effect maturation, which accompanies differentiation in muscle quality is expected to cause performance change CODS.

To examine how age of the players predict RSA performance, the best time of RSA scores (i.e., the best (minimum value among the 6 consecutive sprints) has been taken. GLM was used to test the prediction capacity. This is because that age does not have significant relationship RSA total time, RSA average time or RSA worst time. There was only a significant relationship between age and RSA best time. The model or equation thus is: CODS time $=$ 12.133-0.098(age).

The overall regression model was significant $\mathrm{F}(1,86)=5.01, \mathrm{p}=.028, \mathrm{R}$ squared $=.055$. The coefficient of age is significant, meaning that a unit change in age can cause a 0.30 second change or reduction. The effect of age is higher with that of RSA best time as it is highly dependent on sprinting quality. As players get matured and strong because of indigenous hormone testosterone, they are likely to be good in sprinting and RSA best. The study was done with only male soccer players the effect of maturity or testosterone can immensely matter speed and speed or strength related performance. However, those measures as RSA total time, RSA average time and RSA worst time can rely on other training related qualities like aerobic fitness or lactate threshold. The ability to get rid of lactate which is inherent to anaerobic glycolysis during repeated sprinting, can greatly impact these performance parameters of RSA.

\section{Conclusion}

When maturation comes quality differentiation and performance or fitness increments are highly expected. What this study came across is that players with the higher age tend to perform better when fitness is examined. Linear sprinting speed, CODS and RSA best are physical qualities which are dependent on the age level of the players.

When we evaluate players performance in relation to speed we need to acknowledge and consider the effect of maturity on performance. Therefore, during talent identification, we ought to know that regardless of size less matured or even late matured players may be less advantageous.

Other researchers need to focus on how specific effect of speed or speed related training regimens is affected by maturity level. How the effect of these training protocols is moderated by maturity level. For better elucidating the matter, the question of at which age or maturity level does speed and speed related trainings produce the best possible outcome is worthy of scientific investigation..

\section{References}

[1] J. Bangsbo, M. Mohr, \& P. Krustrup, Physical and metabolic demands of training and match-play in the elite football player, Journal of Sports Sciences, 24 (2006) 665-674.

[2] C. Carling, A. Williams, \& T. Reilly, (2005). Handbook of soccer match analysis: A systematic approach to improving performance, London, Routledge.

[3] M. Millard-Stafford, G.L. Warren, L.M. Thomas, J.A. Doyle, T. Snow, K. Hitchock, Recovery from run training: efficacy of a carbohydrate-protein beverage?, International Journal of Sport Nutrition and Exercise Metabolism 15 (2005) 610-624.

[4] C. Barnes, D.T. Archer, B. Hogg, M. Bush, \& P.S. Bradley, The evolution of physical and Technical Performance parameters in the English Premier League, International Journal of Sports Medicine, 35 (2014) 1095-1100.

[5] P.S. Bradley, C. Carling, A. Gomez Diaz, P. Hood, C. Barnes, J. Ade, M. Mohr, Match performance and physical capacity of players in the top three competitive standards of English professional soccer, Human Movement Science, 32 (2013a) 808-821.

[6] J. Bangsbo, Physiological demands of football, Sports Science Exchange, 27 (2014) 1-6. 
Belayneh Chekle et al., /2019

[7] P.S. Bradley, M.D. Di Mascio, D. Peart, P. Olsen, \& B. Sheldon, High-intensity activity profiles of elite soccer players at different performance levels, Journal of Strength Conditioning Research, 24 (2009a) 2343-2351.

[8] P.S. Bradley, C. Lago-Penas, E. Rey, \& A.G. Diaz, The effect of high and low percentage ball possession on physical and technical profiles in English FA Premier League soccer matches, Journal of Sports Sciences, 31 (2013b) 12611270.

[9] R. Durate, D. Araujo, C. Vanda, \& K. Davids, Sports teams as super organisms: Implications of sociobiological models of behavior for research and practice in team sports performance analysis, Sports Medicine, 42 (2012) 633-642.

[10] W. Frecken, K. Lemmink, N. Delleman, \& C. Visscher, Oscillations of centroid position and surface area of soccer teams in small-sided games, European journal of Sports Sciences, 11 (2011) 215-223.

[11] C. Carling, F. Le Gall, \& G. Dupont, Analysis of repeated high-intensity running performance in professional soccer, Journal of Sports Sciences, 30 (2012) 325-336.

[12] T. Haugen, E. Tonnessen, J. Hisdal, \& S. Seiler, The role and development of sprinting speed in soccer. Brief review, International Journal of Sports Physiology and Performance, 9 (2014) 432-441.

[13] 0. Faude, T. Koch, \& T. Meyer, Straight sprinting is the most frequent action in goal situations in professional football, Journal of Sports Science, 30 (2012) 625-631.

[14] T.J. Gabbett, The training-injury prevention paradox: Should athletes be training smarter and harder?, British Journal of Sports Medicine, 50 (2016) 273-280.

[15] D. Farrow, W. Young, \& L. Bruce, The Development of a Test of Reactive Agility for Netball: A New Methodology. Journal of Science and Medicine in Sport, 8 (2005) 52-60.

[16] J. Sheppard, W.B. Young, T. Doyle, T. Sheppard \& R.U. Newton, An evaluation of a new test of reactive agility and its relationship to sprint speed and change of direction speed, Journal of
Science and Medicine in Sport, 9 (2006) 342349.

[17] P.S. Bradley, W. Sheldon, B. Wooster, P. Olsen, P. Boanas, \& P. Krustrup, High intensity running in English FA Premier League soccer matches, Journal Sports Sciences, 27 (2009b) $159-168$.

[18] J. Bangsbo, F.M. Iaia, \& P. Krustrup, Metabolic response and fatigue in soccer, International Journal of Sports Physiology \& Performance, 2 (2007) 111-127.

[19] M. Beato, M. Bianchi, G. Coratella, M. Merlini, \& B. Drust, Effects of Plyometric and Directional Training on Speed and Jump Performance in Elite Youth Soccer Players, Journal of Strength and Conditioning Research, 32 (2017) 289-296.

[20] Y. Negra, H. Chaabene, J. Fernandez-Fernandez, S. Sammoud, R. Bouguezzi, O. Prieske, \& U. Granacher, Short-Term Plyometric Jump Training Improves Repeated-Sprint Ability in Prepuberal Male Soccer Players, Journal of Strength and Conditioning Research, 1 (2018).

[21] G. Sporis, I. Jukic, L. Milanovic, \& V. Vucetic, Reliability and Factorial Validity of Agility Tests for Soccer Players, Journal of Strength and Conditioning Research, 24 (2010) 679-686.

[22] A.M. Zagatto, W.R. Beck, \& C.A. Gabbetto, Validity of running anaerobic sprint test for assessing anaerobic power and predicting short distance performance, Journal of Strength and Conditioning Research, 23 (2009) 18201827.

[23] B.C. Bongers, M.S. Werkman, D. Blokland, M.J.C. Eijsermans, P. van der Torre, B. Bartels, T. Takken, Validity of the Pediatric RunningBased Anaerobic Sprint Test to Determine Anaerobic Performance in Healthy Children, Pediatric Exercise Science, 27 (2015) 268-276.

[24] W.G. Hopkins, S.W. Marshall, A.M. Batterham, \& J. Hanin, Progressive statistics for studies in sports medicine and exercise science, Medicine \& Science in Sports \& Exercise, 41 (2009) 3-12.

[25] S. Emmonds, S. Scantlebury, E. Murray, L. Turner, C. Robsinon, \& B. Jones, Physical Characteristics of Elite Youth Female Soccer Players Characterized by Maturity Status, 
Belayneh Chekle et al., /2019

Journal of Strength and Conditioning Research, 1 (2018).

[26] M. Di Mascio, J. Ade, C. Musham, O. Girard, \& P.S. Bradley, Soccer-Specific Reactive RepeatedSprint Ability in Elite Youth Soccer Players, Journal of Strength and Conditioning Research, 1 (2017).

[27] M.A. Selmi, B. Al-Haddabi, M.H. Yahmed, \& R.H. Sassi, Does Maturity Status Affect The Relationship Between Anaerobic Speed Reserve And Multiple Sprints Sets Performance in Young Soccer Players?, Journal of Strength and Conditioning Research, 1 (2017).

[28] H. Pojskic, E. Åslin, A. Krolo, I. Jukic, O. Uljevic, M. Spasic, \& D. Sekulic, Importance of Reactive Agility and Change of Direction Speed in Differentiating Performance Levels in Junior Soccer Players: Reliability and Validity of Newly Developed Soccer-Specific Tests, Frontiers in Physiology, 9 (2018).

\section{Funding}

This study was not funded by any grant

\section{Acknowledgements}

The authors would like to thank every participant for his effort and time.

\section{Conflict of interest}

None of the authors have any conflicts of interest to declare.

\section{Informed consent}

All participants gave written informed consent to participate in this study.

\section{About The License}

\section{()ㅜㅇ}

The text of this article is licensed under a Creative Commons Attribution 4.0 International License 